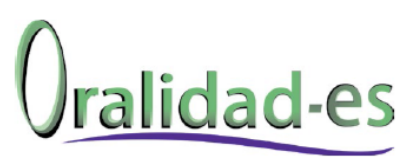

\title{
Una palabra para construir escenarios de aprendizaje y de paz
}

\author{
A word to build scenarios of learning and peace
}

\author{
María Catalina León-Suárez \\ mariacatalinaleonsuarez@gmail.com \\ Universidad de Buenos Aires, Argentina
}

León-Suárez, M. C. (2017). Una palabra para construir escenarios de aprendizaje y de paz. Oralidad-es, 3(6), 38-47.

Fecha de recepción: 20 de julio de 2017 / Fecha de aprobación: 14 de noviembre de 2017 


\section{Resumen}

El artículo "Una palabra para construir escenarios de aprendizaje y de paz", tiene por objetivo reconocer dentro de las reflexiones sobre la oralidad, el valor estructurante de la palabra en el sujeto y en las relaciones sociales, en el ámbito educativo. La palabra como forjadora del sujeto, constructora de escenarios para el encuentro con los otros y creadora de oportunidades para la sana convivencia y la paz. Los conceptos son tomados del Psicoanálisis. Se relatan dos experiencias en el ámbito educativo: una a nivel individual en procesos de enseñanza-aprendizaje y otra en el trabajo con la comunidad.

\section{it Palabras clave}

Palabra; sujeto; relaciones sociales; educación; paz

\section{Abstract}

The article "A Word to Build Scenarios of Learning and Peace", aims to recognize within the reflections on orality, the structuring value of the word in the subject and in social relations, in the educational field. The word as the forger of the subject, builder of scenarios for the encounter with others and creator of opportunities for healthy coexistence and peace. The concepts are taken from Psychoanalysis. Two experiences in the educational field are related: one at an individual level in teaching-learning processes and another at work with the community.

Key words

Word; subject; social relations; education; peace 


\section{Introducción}

Se nos ha invitado a pensar en la oralidad, a reflexionar cómo contribuye ésta en el desarrollo de una cultura de paz, a debatir experiencias, investigaciones, posturas al respecto; es sin duda una invitación atractiva, que implica un doble reto o quizá triple, por un lado, supone asumirse sujeto en cuanto se nos ha otorgado la palabra, por otro, que ésta transmita a la vez una reflexión sobre sí misma, el poder de la palabra, su alcance..., una reflexión que inicia por la palabra que se me ha otorgado y pasa por los efectos que tiene sobre los demás, sobre lo social, aún más, es una invitación a pensar puntualmente su consecuencia sobre la paz, y por eso el reto se vuelve mayor, porque se trata de decir con la palabra lo que está armado por experiencias de vida, por palabras, para desarrollar en el tiempo que incluye el ahora, una cultura de paz.

Asumidos estos retos, es preciso decir que la presente reflexión se inscribe en el ámbito educativo debido al papel trascendente y sin igual que tiene en la sociedad y por ende en cada sujeto. No en menos la escuela es el escenario que por excelencia recibe al sujeto en el mundo de la relación con los otros después del encuentro que ha tenido con su familia, presentando el mundo, la posibilidad de crear, de soñar, de saber cada vez más. Es por esto un escenario rico en experiencias, en encuentro de realidades diversas, en conflictos, en soluciones, en creatividad, y no es redundante decir, es un espacio rico en aprendizaje.

La educación, particularmente la escuela, es un escenario en el que la oralidad es la protagonista, y puede ser máxima herramienta para desarrollar una cultura de paz. Sobre esto pretendemos dar cuenta por medio del relato de dos experiencias, una a nivel de la comunidad en general y otra, en la particularidad del encuentro con el estudiante, en las que la oralidad tiene un lugar fundamental vía el papel estructurante de la palabra en el sujeto y por ende la función simbó- lica que de ahí en más cumple en la relación de éste con los otros y en la posibilidad de generar procesos de aprendizaje y de paz.

Es así como antes de compartir estas experiencias, se hace necesario propiciar una reflexión sobre la palabra, sobre ese lugar fundamental que se le adjudica y que permite generar los procesos mencionados, puesto que el presente escrito se refiere a una hipótesis corroborada en el día a día de la educación, una hipótesis a la que se hace mención cuando inicialmente se dice, nos asumimos sujetos en cuanto se nos ha otorgado la palabra, señalando que es a través del retorno a la palabra verdadera, a esa que se escucha, a esa que se dice, en la profundidad de lo que significa escuchar y decir, que emerge el sujeto y con él, los procesos en los que se entablan lazos con los otros, se construye con el otro, se aprende y desarrolla una cultura de paz.

\section{¿Qué palabra?}

Tempranamente en la obra de Sigmund Freud, creador del Psicoanálisis, y de otros importantes psicoanalistas como Jacques Lacan, se pone en evidencia cómo los seres humanos somos sujetos de lenguaje y perdemos nuestra naturalidad, la condición meramente orgánica, a través del "baño de lenguaje" que recibimos en el encuentro con el otro, con la madre, cuestión que nos diferencia sustancialmente de cualquier otro ser vivo. Aún antes de nacer, somos ya nombrados por los otros, se profieren palabras de alegría, de temor, de expectativa...sobre nuestra venida al mundo, y es por estas palabras que, sin haber acaecido el alumbramiento, ya comenzamos a existir. La interpretación que hace la madre sobre el grito del bebé es uno de los registros primordiales en el que el lenguaje comienza a forjar ese sujeto, cada cuidado proferido por la madre organiza un cuerpo que es aseado a la forma de la cultura, un cuerpo que es cuidado, que es alimentado y al que se le exige una regula- 
ción...de esfínteres, de tiempo de alimentación, de manejo del sueño...es en últimas, el cuerpo forjado por la palabra, atravesado por esta para así hacerse parte del mundo familiar, del mundo de lo social. En esta relación establecida con los otros media entonces el lenguaje, la palabra, que no solo organiza el cuerpo, sino que otorga al sujeto en constitución un lugar en el mundo. Es a través de las palabras que principalmente se pone en evidencia el deseo que movilizó en los padres la venida del niño al mundo, se trata de un deseo que lo captura y le ubica en un lugar a partir del cual comienza a vivir y a asumir la existencia propia; se trata, en este momento trascendental, de que la relación entre la madre y el niño cada vez más no tiene una absoluta compenetración, que la relación con el niño va más allá de ser asistido por lo que al parecer son sus necesidades, una relación que así, es nada más ni nada menos que la que da paso al mundo simbólico que implica la oralidad, la palabra.

Quizá hasta aquí podemos dar cuenta, en cierta medida, del porqué a la palabra le adjudicamos al inicio un lugar trascendente, que va incluso más allá del acto comunicativo, un lugar constitutivo de la subjetividad, pero ¿por qué consideramos este momento descrito como paso al mundo simbólico? y ¿por qué considerar que en esto se encuentran las bases para el aprendizaje y para una cultura de paz?

Esta relación entre la madre y el niño en la que ya no son uno sólo, en la que la madre no se ocupa completamente de interpretar sus necesidades y nombrarlas a su manera, introduce una distancia, una falta entre ambos: que la madre no esté siempre presente implica que desea a ese sujeto niño, pero no sólo a él, hay algo más de lo que se ocupa, algo que captura su deseo, razón por la que aquello con lo que la madre satisface al niño pasa a tener un valor simbólico, el valor de un don, de un objeto de satisfacción que se vuelve signo de su voluntad, signo de su amor, de su presencia pero también de su ausencia.
Las palabras de esta manera, pasan a decir más de lo que en sí mismas pueden significar, son "símbolo de", así como lo son los demás objetos que entre los dos coexisten, hay una falta entre el niño y la madre que implica una madre que desea más allá de ese hijo y un hijo, un sujeto naciente que desea saber qué es aquello que ocupa a la madre, qué es eso que ella desea que no es solamente él, esto es la base de su propio deseo, el de él, y de la relación deseante que entabla desde ahí con los otros, con el saber, con la vida misma.

Sin embargo, lo que puede ocurrir es que esas palabras que estructuran esa subjetividad, no son precisamente aquellas que introducen la falta y con ella el deseo, contrario a ello puede que la madre tenga siempre una interpretación absoluta de lo que el sujeto necesita, que siempre esté presente para colmar estas necesidades, que no haya una falta que dé paso a desear eso que falta. Dos caminos que en últimas hablan de dos lugares distintos en los que puede ser puesto el niño y dos posiciones por las que puede optar así mismo el otro materno. Son dos alternativas que se reproducen de manera similar en las distintas relaciones que se entablan en lo social y de forma clara en la educación.

En una relación entre la madre y el niño que está habitada por la falta, no sólo se ha dado apertura al mundo de lo simbólico, sino que el niño ha sido puesto en un lugar en el que es un sujeto en constitución, diferente a la madre, un sujeto que al no tenerlo todo, de ahí en adelante pasa la vida entera deseando ese todo que nunca va a llegar a ser completo, por el que siempre va a desear saber más, alimentarse más, conocer más...contrario a ello, una relación que no está mediada por la falta es aquella que pone al sujeto en constitución en un lugar de objeto, dado que no hay en él la posibilidad de desear; quien le asiste con sus cuidados permanentes pensará por él, deseará por él, y aquello que dará no será producto de un don de amor, no tendrá una representación simbólica sino será producto de 
una absoluta presencia que no le permite ser. Es a través de este breve recuento de lo que ocurre en la estructuración subjetiva, en el que la oralidad vista como el efecto de la palabra misma, aquella que construye un cuerpo, que otorga un lugar en el mundo, permite pensar esa intervención fundamental del lenguaje y que así como ocurriera en un principio, es una intervención que se reproduce una y otra vez en las relaciones sociales, en la educación, para construir lugares donde los sujetos van a ser ubicados y desde los cuales van a responder.

Es por esta razón que aquí se encuentran unas bases determinantes de los procesos de aprendizaje y los cimientos de una cultura de paz, cuando la palabra de los diferentes actores educativos, la palabra del estudiante, la del maestro... es proferida y escuchada como proveniente de un sujeto y no de un objeto, se crean procesos de aprendizaje, se construye una cultura de paz, por esto es importante pensar ¿qué palabra?, sostenemos la hipótesis de que es necesaria una palabra para construir escenarios de aprendizaje $y$ de paz, pero no cualquier palabra, ¿qué palabra?, aquella proferida con la profundidad de lo que significa escuchar y decir. Es tiempo entonces de presentar dos experiencias a través de las cuales se desarrolla de forma más clara lo expuesto, ahí donde la oralidad, la palabra, cobra un lugar preponderante en la educación porque moviliza la falta, el deseo, devuelve la palabra al sujeto.

Cuando se recupera la palabra, emerge el sujeto del aprendizaje y ello tiene un efecto pacificador. Si hay un hecho que tranquiliza, que posibilita procesos de pacificación para el sujeto, es precisamente aquel que crea las condiciones para que haya un encuentro con los otros, la posibilidad de estar en un lugar, de hacer parte de éste de una forma que resulte menos perjudicial para sí mismo. Y es que hay distintas formas de estar y de hacer parte de un lugar, pero muchas veces éstas van en detrimento de la oportunidad de crecer, de aprender, de encontrarse con los otros, de ser sujeto.
Es así que en mi trabajo profesional como psicopedagoga he tenido que encontrarme, en varias oportunidades, con niños que no han conseguido una manera distinta para estar en la institución escolar que siendo el niño problema, el que no se está quieto o el que no aprende. En muchas ocasiones este lugar, nada halagador ni tranquilizador, que ocupan los estudiantes, pende de la "palabra certera" de quienes están a su alrededor y "conocen bien" de sus dificultades.

Lorena, por ejemplo, es una niña de trece años que desde muy temprana edad se encuentra en una fundación, cuya misión es atender niños con distintos tipos de adicción, de ella se dice jocosamente "hace parte del inventario de la institución”, es decir, de todos los bienes y activos con que se cuenta, puesto que desde muy temprana edad ha vivido allí y aunque ha habido intentos de marcharse, siempre termina volviendo; quizás esta es la razón por la que en aquel lugar todo se sabe de ella: de dónde viene y por qué, qué le gusta, qué puede y no hacer, entre lo cual está muy claro, no puede aprender. Las maestras relatan una y otra vez la imposibilidad de que aprenda al menos a escribir su nombre y la psicóloga, por su parte, ha practicado los respectivos test de coeficiente intelectual que reiteran, en una palabra, la imposibilidad.

Hasta aquí podría decirse, como ocurriera en un primigenio encuentro no mediado por la falta entre la madre y el niño, que hay unas palabras que dan existencia en este momento a Lorena: son certeras y le otorgan un lugar en el mundo, el lugar de no saber, de no aprender, de no tener una palabra distinta sobre sí que no sea la que el ámbito educativo y científico ha dictaminado.

Son palabras con las cuales es nombrada y no faltan en nada, por cuanto tienen ese saber completo sobre ella, el saber sin falta que también considera tener la madre sobre las necesidades del niño. 
Como es de esperarse en una primera entrevista con Lorena, lo que ofrece como carta de presentación es "no sé escribir, no sé leer y no puedo aprender", ante la solicitud de que tome la hoja y el lápiz y escriba lo que se le diga, señala que lo hará porque se lo piden, pero advierte, nada estará bien, por supuesto lo confirma cuando confunde las letras en las palabras, omite otras, o simplemente no sabe qué imprimir en el papel.

Lo que continúa es un no saber sobre el historial de aprendizaje de Lorena y dejar de lado las estrategias establecidas, esperables, que le permitirían acceder a la lectura y a la escritura para construir otras con ella y quizá en un futuro retomarlas, pero para ello se requiere de su asentimiento, una palabra verdadera, que provenga de ella, y que no sea la de los otros que han pronosticado sobre su no posibilidad de aprender.

Es la razón por la que reitero a Lorena que escriba su nombre, a lo cual me responde, ¿es que acaso usted no sabe que yo no sé escribir?, y yo confieso, "no, no sé... ¿no sabe escribir o no quiere escribir?”. Es a través de esta intervención, de mis primeras palabras proferidas sobre ella, que busco ubicarla en un lugar distinto al que había sido ubicada e intento devolverle la palabra, que ella pueda recuperarla para que emerja el sujeto del aprendizaje, pues ¿quién podría saber mejor de su historia de aprendizaje, de sus posibilidades de aprender, sino ella misma?

$\mathrm{Su}$ respuesta es sorprendente, su rostro toma un color rojizo, titubea, alega que no sabe escribir, ni leer, ni sumar, ni restar...después de un tiempo toma la hoja, el lápiz, y con una letra patoja escribe: "Lorena", se levanta y se va. Alcanzo a vislumbrar algo distinto en su rostro, ¿podrá ser el efecto de la palabra sobre el cuerpo?

Con alegría tomo esta escritura como una seña de su ser, un asentimiento que proviene de ella y que le traslada la palabra, la posibilidad de decir sobre su proceso y de tomarlo, la palabra no es en este momento mía como profesional en educación que sabe bien cómo se debe aprender, no lo sé todo sobre Lorena, por lo cual estoy en falta, y al estarlo, deseo saber sobre ella, deseo acompañar la construcción de un camino que le permita aprender, que le permita desear saber.

¿Qué es más simbólico que los procesos de aprendizaje mismos?, la adquisición del lenguaje escrito, a ello en este momento se enfrenta esta niña, no es posible responder con un saber acabado y único de cómo se escribe, se lee, se suma; en los procesos de enseñanza aprendizaje también se pone en juego un don de amor, se dona una palabra que no existía, como quizá hizo Lorena al dejarla sobre mi puesto; se dona un no saber para poder saber, como hice en mi encuentro con Lorena, el maestro y el aprendiz en una relación mediada por el amor al saber, por el deseo de saber. Lo que sigue con Lorena es, cada vez más, un continuo coqueteo con los espacios de lectura y de escritura existentes, así en principio no haga nada distinto a escuchar, algo que antes solo se permitía por obligación. Empieza a asistir a espacios de aprendizaje creados para ella, unas veces más a gusto que otras...lo que no se puede negar es un rostro distinto, una postura diferente sobre su proceso...no es solo su rostro, es el de los demás, que ahora extrañamente no dicen, observan. A veces se escucha a Lorena discutir con sus compañeros, ellos buscan burlarse de su no saber sobre las letras, sobre los números, ella con seguridad les responde que sí sabe.

En este encuentro con la particularidad de un sujeto podemos reconocer la importancia sin igual de la palabra, de la oralidad. La palabra crea lugares, da existencia; respecto a una cultura de paz, es a través de lo que se profiere y de la capacidad de escucha, que se da un lugar a la subjetividad con la complejidad que esta comporta, y esto es, en últimas pacificador; más allá del lugar que socialmente ocupemos, el de saber, el del poder...hay siempre un sujeto con el cual relacionarse, no un objeto de nuestro saber, y es por excelencia ello, el respeto por el sujeto que tenemos al frente, lo que desarrolla escenarios de paz. 


\section{Cuando se recupera la palabra se nombran espacios para la paz}

Tuve la fortuna de participar durante algunos años en un proceso con las instituciones educativas públicas de la ciudad de Bogotá, cuyo propósito era construir con la comunidad educativa una red de escenarios de participación a través de los cuales se generaran procesos de convivencia y de formación ciudadana. Es en este contexto que acompaño, tras el objetivo, a tres instituciones ubicadas en lugares con altos índices de deserción escolar, violencia, homicidios, robos, consumo de sustancias psicoactivas, y es precisamente en este escenario donde puedo reconocer con claridad el poder creador y transformador de la palabra, aquel que propicia encuentros que marcan la vida de las personas y que pueden llegar a suponer una forma distinta de ver y asumir la existencia.

En una de estas instituciones logra reconocerse que existen fuertes dificultades de convivencia no sólo al interior del colegio sino alrededor de éste, especialmente relacionadas con el consumo y el expendio de sustancias psicoactivas, que como es de suponerse, afecta en últimas a la institución. Tras reconocer esta dificultad los directivos del lugar asumen el reto de ceder la palabra al estudiantado y a los maestros, de no generar acciones, como en muchas ocasiones ocurre, en las que las decisiones y las iniciativas son tomadas únicamente por ellos como cabeza administrativa de la institución, para lo cual se convoca a algunos líderes estudiantiles, maestros, orientadores y coordinadores a una reunión en la cual se toma una decisión trascendental.

Entre todos, pero especialmente con las ideas que aportan los estudiantes, se ha resuelto emprender un proceso para generar mayor participación de la comunidad: la realización de un festival en el que se haga una toma simbólica del parque que se encuentra afuera del colegio, para nombrarlo de una forma distinta, no el parque del consumo, del robo, sino el parque de la cultura, de la convivencia y la paz; una oportunidad en la que además pueda nombrarse de manera diferente por la comunidad del barrio la institución, no como el colegio de los vagos, sino el colegio donde pasan cosas importantes, donde hay danza, música, deporte, saber.

El camino se emprendió y puede decirse que trajo grandes sorpresas consigo, no sólo los directivos, los apasionantes maestros y los inquietos líderes estudiantiles hicieron parte de la iniciativa, literalmente el festival traspasó los muros del colegio, la casa de la cultura juvenil con hip-hop, break dance, y rock quiso unirse a la jornada, así como la acción comunal representada por los ancianos con sus danzas de folclor tradicional, algunos jóvenes del barrio que ya no estudiaban en el colegio, padres de familia que hicieron "aguapanelita" e incluso, el señor de la panadería que llevó algunas bolsas del manjar para compartir.

La fiesta del gran día incluyó no sólo estas muestras, los niños del jardín, algunas chicas de primaria, así como el grupo institucional hicieron presencia con las danzas, el profe de teatro se animó a presentar su monólogo, la profe de educación física a hacer los aeróbicos con los que se inició la jornada, los proyectos de juegos tradicionales y deportes trajeron las actividades que se llevaron a cabo simultáneamente a las que se presentaban en la tarima, el rector lideró las palabras de reflexión sobre la convivencia, la juventud las cantó, las danzó, las escribió y las dibujó...estudiantes con gusto por el dibujo y el grafiti se tomaron los muros del colegio al que nombraron espacio para la paz y la sana convivencia, los estudiantes del semillero de periodistas estuvieron a cargo de toda la presentación del festival, entrevistaron padres, estudiantes, transeúntes del parque. 
Pero todo esto fue producto de un arduo proceso en el que hubo que ceder la palabra a la comunidad, a los estudiantes y a la juventud en general, que muchas veces es acallada por el adulto con las normas que no logran tener un sentido para ellos, con programas de estudio estrictamente encuadrados que no permiten otras formas, otras posibilidades, con el uso de la palabra sólo cuando les es permitido, con la imposibilidad de participar en decisiones que competen la convivencia y a su colegio en general; cuando esto ocurre el estudiante, el maestro, no es reconocido en su subjetividad, es un objeto más del sistema al que se le ha quitado la palabra y la escuela pasa a ser un lugar que difícilmente transmite vida, pasión por el saber, para convertirse en un escenario en el que el aburrimiento, el desinterés y lo que desencadenan éstos son un grito sordo para recuperar la palabra.

Tuve la grandiosa oportunidad de ver los efectos que este proceso produjo en la comunidad, en el sujeto estudiante, que la palabra les fuera devuelta, que hubiese una sincera apertura a su decir y a la escucha. Como nada estaba establecido y todo por construir, cada uno buscó qué hacer: los estudiantes que iniciaban el bachillerato se encargaron de hacer cientos de tarjetas de invitación a las familias, con su puño y letra, los amantes del hip hop y el rap no podían creer que iban a tener un espacio para decir a través de su melodía y los practicantes del break dance tampoco creían que la institución compraría los anhelados tapetes para su práctica, los líderes estudiantiles y los grados once tuvieron a su cargo todo el montaje logístico, desde el orden de las presentaciones hasta la estrategia de seguridad. Se hicieron carteles que se pegaron por los barrios invitando a la comunidad y un día antes, con megáfono en mano, recorrieron las calles anunciando el evento.

Es cierto, justo aquellos estudiantes que faltaban con frecuencia a clase, que estaban perdiendo el año escolar, eran los que más animados hacían parte de la organización, varios de los jóvenes que frecuentaban el parque, de los cuales se decía consumían sustancias psicoactivas en él y delinquían de otras maneras, se quedaron allí, observaron, pudieron compartir el gusto por la música y durante la jornada no hubo consumo, algunos eran exalumnos de la institución y parecían tener un reencuentro con la misma. Recuerdo aún la confesión que me hizo un estudiante días después: "que el profe me haya llamado para que pintara, para que me expresara con mis dibujos me dio un sentido, entendí para qué estoy aquí, para qué sirvo y no se me va a olvidar”.

He aquí una experiencia desde el encuentro con la comunidad educativa y con cada sujeto. Aquí un mensaje similar al que encontrábamos en la experiencia con Lorena, la importancia de recuperar la palabra, pues con tan sólo este hecho se crean nuevas oportunidades, nuevos lugares que habitar, pero la pregunta una vez más insiste: ¿qué palabra? Podemos recordar nuevamente el valor simbólico y lo que significa, sí, esto nos lleva una vez más al encuentro primordial con el otro materno, que la presencia existe bajo el fondo de la ausencia y que es esto lo que habilita el deseo...de saber, de aprender, de participar, de hacer parte de...La escuela no puede ser absoluta presencia, un bloque terminado: con reglas construidas por pocos e inamovibles, con contenidos escolares estructurados meticulosamente, acabados y rígidos, es necesario que haya cabida a la ausencia, a que no todo esté dicho y hecho porque es a través de esto que se moviliza el deseo y con él se recupera la palabra, emerge la subjetividad.

Los estudiantes y maestros de esta institución así nos lo dejan ver con claridad, y nos muestran algo extraordinario, el deseo es prendedizo, se prende por contagio...gran descubrimiento, especialmente en procesos que requieran hacer lazo social, anudar fuerzas y sobre todo cuando de una sana convivencia y de paz se habla. Esta experiencia nos deja otra reflexión más, y en ello me permito citar al pedagogo Jorge Larrosa (in- 
édito): "las palabras dicen lo que dicen y además más y otra cosa”, el colegio fue rebautizado con unas nuevas palabras, y para prevenir el olvido, estas palabras se escribieron en sus muros por los estudiantes y al modo de ellos: "Colegio un espacio de paz y sana convivencia”, pero no basta con el "nuevo nombre", éste dice además más y otra cosa, dice de una experiencia en la que se recobró la palabra, dice de las nuevas palabras que es necesario continuar profiriendo, de que no todo está dicho ni va a estarlo, pero que es necesario nombrar para crear espacios para la paz.

\section{A modo de conclusión}

Relatar lo que significa para mí la oralidad, la palabra en mi ejercicio profesional con la educación, me ha conducido inexorablemente a compartir experiencias que han tocado las fibras de mi subjetividad, que me han devuelto la palabra, incluso para decidir que por momentos es mejor callarla. A través de las experiencias, como las vividas con Lorena y con esta recordada institución, puedo decir que es con la oralidad, con la palabra -no cualquiera-, que se construyen escenarios de aprendizaje y de paz, sólo por una razón, porque con esta palabra hay respeto por el otro, por la subjetividad.

Jorge Larrosa señala:

Tal vez por eso se trata de mantener la experiencia como una palabra y no hacer de ella un concepto, se trata de nombrarla con una palabra y no de determinarla con un concepto. Porque los conceptos dicen lo que dicen, pero las palabras dicen lo que dicen $y$ además más y otra cosa. Porque los conceptos determinan lo real y las palabras abren lo real. Y la experiencia es lo que es, y además más y otra cosa, y además una cosa para ti $y$ otra cosa para mí, $y$ una cosa hoy y otra mañana, y una cosa aquí y otra cosa alli, y no se define por su determinación sino por su indeterminación, por su apertura. (Larrosa: inédito).

Yo, al mejor estilo de Larrosa, busco transmitir esta, mi experiencia, las palabras de lo que me inspira pensar la oralidad con la expectativa de lo que puede producir en los otros, pero especialmente con su indeterminación, la que me garantiza que no todo lo haya dicho, que hay un lugar para el deseo, una apertura que devuelva la palabra al lector, al escucha, y que en ese movimiento se produzca un contagio.

\section{Referencias}

Lacan, J. (1988). Dos notas sobre el niño. En J. Lacan (ed.) Intervenciones y textos II. (1969). Buenos Aires : Editorial Manantial.

Lacan, J. (1999). El reverso del Psicoanálisis. En J. Lacan (ed.) El Seminario, Libro 17 (1969-1970). Buenos Aires: Editorial Paidós.

Lacan, J. (2002). Escritos 2(1901-1981). Buenos Aires: Editorial Siglo XXI.

Larrosa J. La experiencia y sus lenguajes. $23 \mathrm{de}$ noviembre de 2013. Recuperado de http:// www.bnm.me.gov.ar/giga1/documentos/ EL001417.pdf

Laurent E. El niño y su madre. 25 de febrero de 1984. Recuperado de http://es.scribd.com/ doc/91228752/Eric-Laurent\#scribd 